\title{
A colloidal model system with tunable disorder: Solid-fluid transition and discontinuities in the limit of zero disorder
}

\author{
C. Richter ${ }^{1}$, M. Schmiedeberg ${ }^{2, a}$, and H. Stark ${ }^{1}$ \\ 1 Institut für Theoretische Physik, Technische Universität Berlin, Hardenbergstr. 36, D-10623 Berlin, Germany \\ 2 Institut für Theoretische Physik 2: Weiche Materie, Heinrich-Heine-Universität Düsseldorf, D-40204 Düsseldorf, Germany
}

Received 10 May 2011

Published online: 10 October 2011

(C) The Author(s) 2011. This article is published with open access at Springerlink.com

\begin{abstract}
We study a colloidal model system where disorder can be continuously tuned from no disorder - corresponding to a system that can crystallize - to large disorder where geometrical frustration occurs. The model system consists of colloidal particles with screened electrostatic repulsion. They can only move on single lines which are parallel and equidistant to each other. We introduce disorder by modulating the particle line density. The system exhibits a solid-to-fluid transition which we study by the structure factor and the temporal evolution of the mean-square distance of nearest neighbors on neighboring lines. A determining feature is the occurrence of discontinuities when disorder is tuned to zero. We observe that the peak height of the pair correlation function in the solid phase does not extrapolate to the value of the perfect crystal. Similarly, the mean interaction energy and the screening length at which the solid-fluid transition occurs seem to be discontinuous when the limit of zero disorder is approached.
\end{abstract}

\section{Introduction}

In a geometrically frustrated system crystallization is prevented for geometrical reasons. Even in thermal equilibrium, the system is trapped in a highly degenerate disordered ground state which leads to complex phases as can be observed, e.g., in magnets [1], ferroelectric materials [2], water ice [3], ceramics [4], or high-temperature superconductors [5]. Probably, the most famous example of a geometrically frustrated system consists of spins on a triangular lattice that try to form anti-ferromagnetic order [6]. Such so-called spin glasses became a standard example to study structural and dynamical properties of disordered systems. A similar frustration has been observed in buckled colloidal monolayers [7]. Spin-ice-like behavior in a colloidal system can also be achieved by trapping colloids in a special arrangement of optical double-well traps [8]. Furthermore, on a curved surface, disks usually cannot crystallize into a triangular phase and therefore highly degenerate disordered phases occur $[9,10]$. A frustrated solid phase also displays a transition to a fluid. How this transition crosses over into the perfect crystal-to-fluid transition when frustration is reduced, is an interesting and open problem. It is important for many frustrated systems, e.g., for disks on curved surfaces in the limit of small curvature.

Here we introduce a colloidal model system whose disorder can be continuously tuned by a control parameter.

\footnotetext{
a e-mail: schmiedeberg@thphy.uni-duesseldorf.de
}

It is able to cross over from a system which is allowed to crystallize to one with a disordered ground state due to geometric frustration. This enables us to study dynamical and structural properties of the model system, such as pair correlation functions and mean-square displacements, under various degrees of disorder. Interestingly, these properties do not simply extrapolate from the disordered into the crystalline phase when disorder is reduced to zero.

We consider a two-dimensional system of charge-stabilized colloidal particles which can only move on parallel and equidistant lines. The colloids interact according to a repulsive screened Coulomb potential. Since the colloidal particles are not allowed to jump from one line to another, the number of particles on each line is conserved. The mean density is chosen such that in the limit of strong repulsion or large screening length the colloids form a triangular crystal if the number density on all lines is the same. However, if the ratio of number densities from neighboring lines is irrational, a periodic structure is not possible and the colloids are trapped in a geometrically frustrated state. Therefore, by varying the colloidal number densities, we are able to control whether the system crystallizes or assumes a frustrated state (cf. fig. 1). By decreasing the range of the electrostatic interaction, colloids from neighboring lines are able to pass each other and the system displays a solid-to-fluid transition also within the disordered phase. In this paper, we will study this transition by investigating the structure factor, the mean interaction energy, and the temporal evolution of particle distances. 

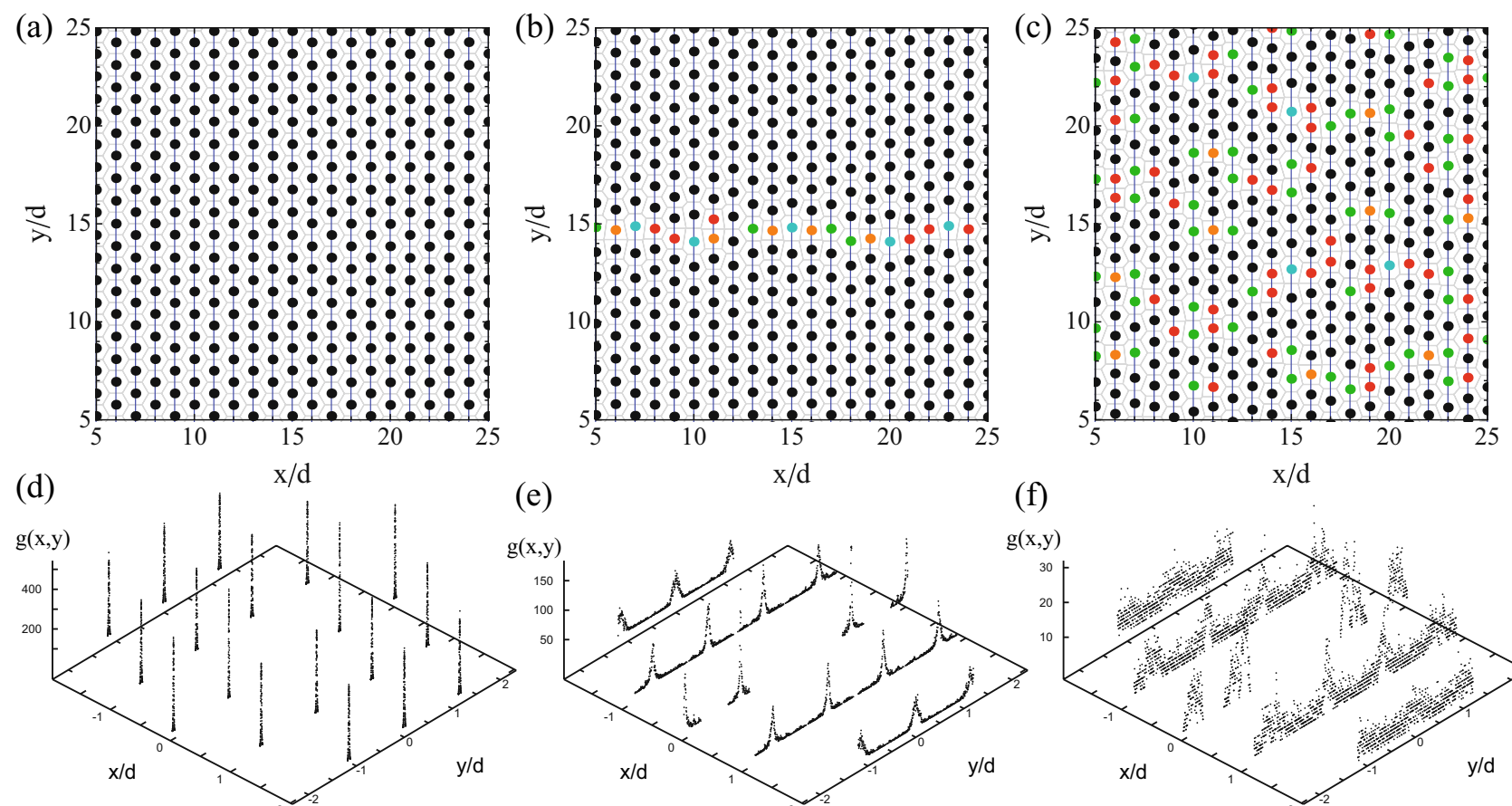

(e)

(f)
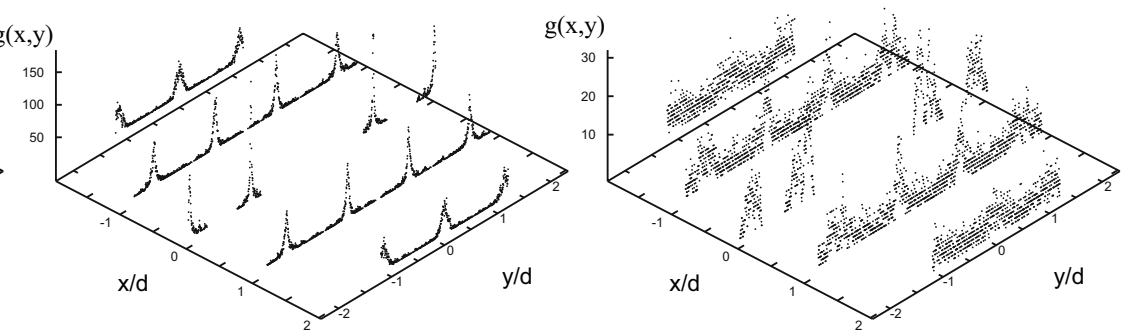

Fig. 1. (Color online) (a) When the colloids occupy all lines with an equal number, they form a triangular crystal. (b,c) The colloidal line density differs from line to line and is aperiodic. Hence, the colloidal particles cannot order periodically. For low disorder (b) defects localized in horizontal ribbons across the lines occur, whereas for larger disorder (c) triangular ordering is hardly visible any more. The number of nearest neighbors is indicated by the color of a particle: black denotes 6 neighbors, red 5 , orange 4 , green 7 and cyan 8 . The lines on which the colloids move are shown in blue. The Voronoi cells of the particles are depicted in gray. (d-f) Pair correlation function $g(x, y)$. (d) For the triangular crystal in (a), (e) for the phase with defects in (b), and (f) for the disordered phase shown in (c). The inverse screening length $\kappa$ in all cases is $\kappa d=1.5$ where $d$ is the distance between neighboring lines. The parameter $A$ introduced in eq. (3) to quantify disorder is 0 in (a,d), 0.033 in (b,e) and 0.149 in $(\mathrm{c}, \mathrm{f})$.

At a first glance, the external control of the number density on each line seems artificial. However, one can think of a system where the external potential consists of parallel one-dimensional potential wells with different depths. At low potential strength, the colloids can cross the barriers between the wells and the number densities in equilibrium depend on the depths of the wells. By increasing the depths of the potential wells, the colloidal particles can no longer jump from one well into another and we obtain our model system from a system with quenched disorder in a random potential. It is possible to realize the system experimentally. For example, one can confine colloidal particles to potential wells created by fast scanning laser beams along lines. Another possibility is the interference pattern of two laser beams with incommensurate wavelengths. The pattern creates a potential landscape for the colloids, where the distances between the wells and their depths follow a one-dimensional quasicrystalline sequence $[11,12]$. For small potential strengths, when the colloids can easily jump from one well into another, periodic phases are still possible. However, for large strengths of the quasicrystalline potential, a disordered frustrated phase occurs. A similar system consists of the vortices in a type-II superconductor where an external potential can, e.g., be realized by magnetic fields [13-15].
If a two-dimensional colloidal suspension is placed into a one-dimensional periodic potential, the particles are usually equally distributed along the lines where the potential is minimal. If such a potential acts on the fluid phase close to the transition into a crystal, the crystalline phase can be induced by increasing the strength of the potential $[16,17]$. For even higher potential strength, the induced crystal may melt again $[18,19]$. This phase behavior can be explained by considering the elastic properties, the fluctuations, and the defects in the colloidal system [20,21]. Note that the defects in a system with homogeneously distributed colloids are thermally induced and therefore are created at high temperatures as pairs of dislocations which unbind at even higher temperature. In our system, where the number density in neighboring potential wells can be different by construction, the defects correspond to vacancies or interstitials and occur even at small temperatures for $A>0$. Therefore, the disordered solid for $A>0$ in our system is different from the smectic phases described in $[20,21]$ where the colloids are homogeneously distributed. Furthermore, reentrant melting at very high potential strength does not occur in our system, because we do no allow the colloids to fluctuate perpendicular to the lines which is required to observe reentrant melting $[20,21,19]$. The phase behavior in a periodic potential 
was also studied for incommensurate densities [22,23]. In our system we keep the mean density constant such that for $A=0$ the density is always commensurate to the line spacing and the colloids can crystallize into a perfect triangular crystal.

In sect. 2 we introduce our model system and comment on details of the simulations. We analyze the structure of the observed phases in sect. 3, determine the mean values of their interaction energies per colloid in sect. 4, and study their dynamics in sect. 5 , in particular the temporal evolution of particle distances. We use the results from the dynamic studies to construct the phase diagram presented in sect. 6. Finally, we conclude in sect. 7 .

\section{Model and simulation details}

We consider colloidal particles that can only move on lines which are arranged parallel to each other with a distance $d$ between neighboring lines. The colloids are charged and interact according to the pair potential of the DerjaguinLandau-Verwey-Overbeek theory [24,25],

$$
\phi(r)=\frac{\left(Z^{*} e\right)^{2}}{4 \pi \epsilon_{0} \epsilon_{r}}\left(\frac{e^{\kappa R}}{1+\kappa R}\right)^{2} \frac{e^{-\kappa r}}{r}
$$

where $r$ is the distance between two colloids, $\kappa$ the inverse Debye screening length, $\epsilon_{r}$ the dielectric constant of water, $R$ the radius of a colloid, and $Z^{*}$ its effective surface charge.

We perform Brownian dynamics simulations for the position $y^{(j)}(t)$ of a colloid on the line with index $j$. It obeys the overdamped Langevin equation

$$
\gamma \frac{\mathrm{d}}{\mathrm{d} t} y^{(j)}(t)=-\sum_{\mathbf{r}^{\prime}} \frac{\partial}{\partial y} \phi\left(\left|\mathbf{r}-\mathbf{r}^{\prime}\right|\right)+f_{T, y}(t) .
$$

Here, $\gamma$ is the friction coefficient, the sum is over all particles with positions $\mathbf{r}^{\prime}$ within a cutoff radius of $5 d$, and $f_{T, y}$ is the thermal random force with zero mean $\left\langle f_{T, y}(t)\right\rangle=0$ and variance $\left\langle f_{T, y}(t) f_{T, y}\left(t^{\prime}\right)\right\rangle=2 \gamma k_{B} T \delta\left(t-t^{\prime}\right)$, where $T$ is temperature. In our simulations, we set $R / d=0.178$, $Z^{*}=7800, \epsilon_{r}=78$, and $T=298.15 \mathrm{~K}$, and use the inverse screening length $\kappa$ as parameter to control the strength of the particle repulsion.

The share of particles that occupies line $j$ at position $x_{j}=j d$ is given by the function

$$
n_{j}=\frac{1-A \sin (j k d)}{\sum_{j^{\prime}}\left[1-A \sin \left(j^{\prime} k d\right)\right]} .
$$

Here $A$ is the parameter that controls the amount of disorder in the system and the wave number $k$ is chosen such that $n_{j}$ never repeats, i.e., the wavelength $2 \pi / k$ is incommensurate to the line spacing $d$. In the following we employ $k=2 \pi /(\tau d)$, where $\tau=(1+\sqrt{5}) / 2$ is the number of the golden mean. In our simulations, $N$ particles are placed on the lines by first distributing colloids according to the integer parts of $N n_{j}$. Then the remaining colloids are successively placed on the lines with the largest noninteger parts of $N n_{j}$. The total number of colloids $N$ is chosen such that they can form a perfect triangular lattice if $n_{j}$ is the same for all lines.

For $A=0$ the particle density on all lines is the same and a transition from a fluid to a crystalline phase occurs when the inverse screening length $\kappa$ decreases. For $A>0$ the density differs from line to line, is aperiodic, and the particles cannot form a crystalline structure.

To achieve an equilibrium state, the system is first prepared at a very large inverse screening length $\kappa d=30$ such that the particle interactions are small and the particles on neighboring lines can easily pass each other. The inverse screening length is then slowly decreased until the final value of $\kappa d$ is reached. Before any structural or dynamical data is taken, the system is simulated for an additional time $t=\gamma d^{2} /\left(k_{B} T\right)$ to ensure equilibration. The time steps of the simulations are chosen between $\Delta t=5 \times 10^{-5} \gamma d^{2} /\left(k_{B} T\right)$ and $\Delta t=10^{-4} \gamma d^{2} /\left(k_{B} T\right)$, depending on the interaction strength. We use periodic boundary conditions and the simulation box usually contains at least $N=400$ particles. The mean particle distance $2 d / \sqrt{3}$ is chosen such that without disorder $(A=0)$ a perfect hexagonal ordering occurs. To exclude possible finite-size effects, we checked that further increase of the system size did not change our results.

\section{Structure}

We determined the pair correlation function $g(x, y)$ as well as the structure factor for different values of the inverse screening length $\kappa d$ and the parameter $A$ used to control disorder in the system. In fig. 1 snapshots and the corresponding pair correlation functions are shown. For $A=0$ (fig. 1(a,d)) the line density is equal on all lines and a crystalline structure is observed. For non-zero $A$, disorder is introduced into the system and the line density varies from line to line. As demonstrated in fig. 1 for $A=0.0333$ (fig. 1(b,e)) and $A=0.149$ (fig. 1(c,f)), the peaks of the pair correlation function become lower and broader when disorder increases with $A$. In fig. 2 the peak height of the maximum, which describes the correlation of neighboring particles on the same line, is plotted for constant $\kappa d=1.5$ as a function of the control parameter $A$. Interestingly, for $0<A<0.1$ the peak height is approximately linear in $A$. However, linear extrapolation to $A=0$ does not reproduce the peak height of the crystalline order at $A=0$. Therefore, the peak height seems to change discontinuously between the crystalline and disordered solid phase. For $A>0.1$, the peak height reaches a constant small value indicating a remaining weak local order as in the fluid phase.

We also analyzed the height of the first maximum in the structure factor, which is a measure for how well periodicity along a line is developed. In fig. 3 we show the peak heights plotted against the inverse screening length $\kappa d$ for different values of $A$. For small $A$ we observe a clear transition from a solid phase at small $\kappa d$, where there is a pronounced peak in the structure factor, to a fluid phase 


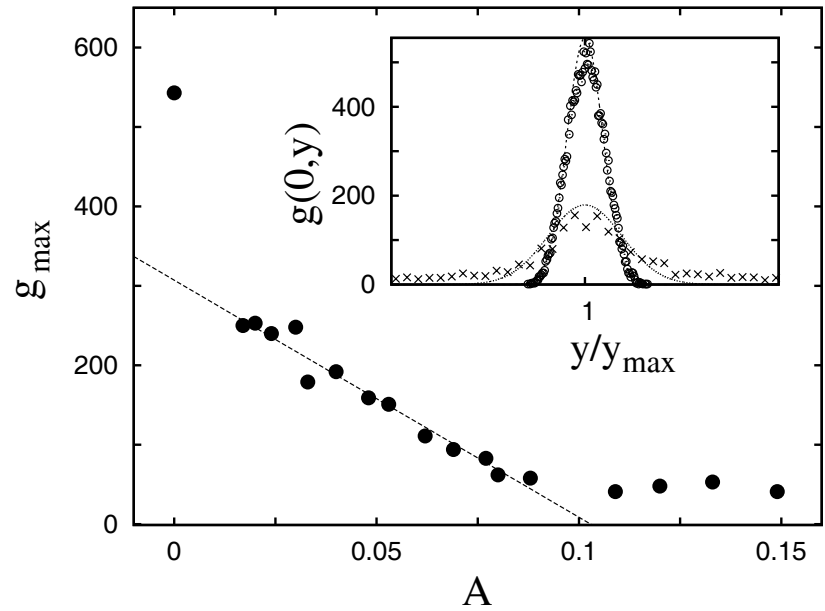

Fig. 2. Height $g_{\max }=g\left(0, y_{\max }\right)$ of the leading maximum of the pair correlation function at $y_{\max }=2 d / \sqrt{3}$ plotted against the control parameter $A$ for an inverse screening length $\kappa d=$ 1.5. The inset compares the corresponding peaks for $A=0$ (circles) and $A=0.0333$ (crosses) to each other.

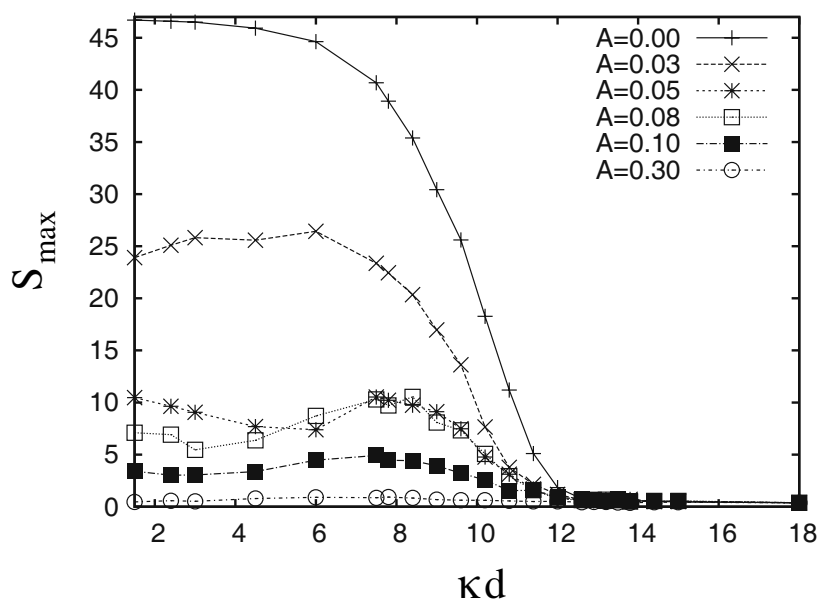

Fig. 3. Height $S_{\max }$ of the first maximum of the structure factor plotted against the inverse screening length $\kappa d$ for different values of the control parameter $A$.

at high values of $\kappa d$. Here, only the typical local order of a fluid remains leading to a nearly vanishing $S_{\max }$. The same small value for $S_{\max }$ is observed for $A>0.1$ for all $\kappa d$. This means that any periodic ordering has vanished in the disordered solid phase (at small $\kappa d$ ) and that its local order is indistinguishable from a fluid (at large $\kappa d)$. Therefore, the solid-fluid transition for large disorder cannot be identified by static quantities such as the pair correlation function or the structure factor. Instead, in sect. 5 we will introduce and study a dynamic criterion based on the temporal evolution of particle distances.

\section{Mean interaction energy}

A further interesting quantity that helps to characterize the different phases of our model system is the mean value
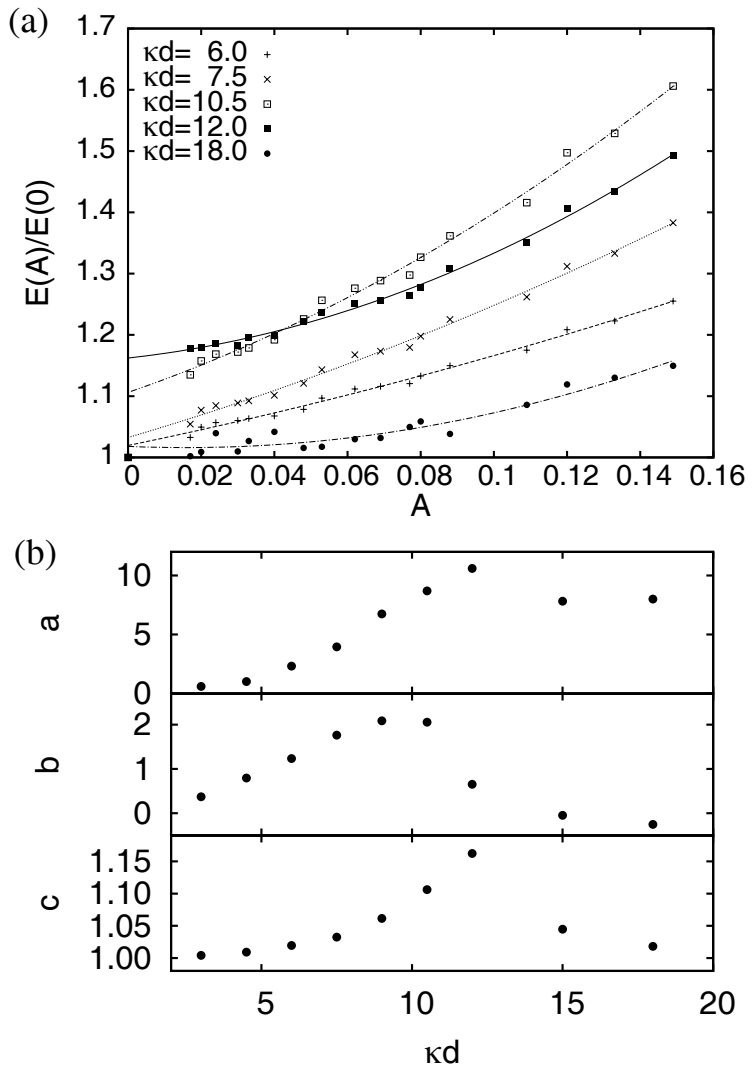

Fig. 4. (a) Mean interaction energy per particle $E$ as a function of the control parameter $A$ for different inverse screening lengths in systems with $N=1024$ colloids. The energies are normalized to the values $E(A=0)$ for each $\kappa d$. Note that, in general, the curves do not extrapolate to 1 for $A \rightarrow 0$. The lines are fits to the quadratic polynomial $a A^{2}+b A+c$ with fit parameters $a, b$, and $c$. The energy increases linearly in $A$ for small $\kappa d$ (solid phase) and purely quadratic for large $\kappa d$ (fluid phase). (b) Fit parameters $a$ and $b$ quantifying, respectively, the quadratic and linear parts of the fitting curves in (a). The third fit parameter, $c$, denotes the energy difference between the system without disorder $(A=0)$ and the extrapolation $A \rightarrow 0$ from systems with $A>0$.

of the interaction energy $E(A)$ per colloid. Figure $4(\mathrm{a})$ plots $E(A)$ as a function of $A$ for different values of the inverse screening length $\kappa d$. We fitted quadratic polynomials of the form $a A^{2}+b A+c$ with fit parameters $a, b$, and $c$ to the energy curves. Figure 4(b) shows the fitting parameters as a function of $\kappa d$. For small values of $\kappa d$, when the system is solid, the energy increases linearly in $A$ meaning $a \approx 0$ in fig. 4(b). However, in the fluid phase at large $\kappa d$, there is a pure quadratic dependence on $A$ as demonstrated by $a>0$ and $b \approx 0$ in fig. 4(b). As we will show in sects. 5 and 6 , for intermediate $\kappa d$ the system is solid for small $A$ and fluid for large $A$. Therefore, a quadratic $(a>0)$ as well as a linear $(b>0)$ contribution to the energy $E(A)$ exists.

The quadratic dependence of $E(A)$ in the fluid phase (large $\kappa d$ ) can be explained as follows: For weak interactions between the colloids, neighboring lines are basically 
decoupled and slide past each other. Then, the total mean interaction energy should mainly be additive in the interaction energies $E_{j}$ for the different lines $j$. We expand $E_{j}$ around the mean value $\rho$ of the line density realized at $A=0$ :

$$
E_{j}\left(\rho+\Delta \rho_{j}\right)=E(\rho)+a \Delta \rho_{j}+\frac{1}{2} b \Delta \rho_{j}^{2}+O\left(\Delta \rho_{j}^{3}\right),
$$

where $\rho+\Delta \rho_{j}$ is the line density at $A>0$ and $\Delta \rho_{j}$ assumes positive and negative values. Since the total number of particles is fixed, we have $\sum_{j} \Delta \rho_{j}=0$. Therefore, in the total mean interaction energy of decoupled lines the linear terms in $\Delta \rho_{j}$ vanish and one arrives at

$$
\begin{aligned}
E(A)= & \sum_{j} E_{j}\left(\rho+\Delta \rho_{j}\right)= \\
& N_{L} E(\rho)+\frac{1}{2} b \sum_{j} \Delta \rho_{j}^{2}+O\left(\sum_{j} \Delta \rho_{j}^{3}\right),
\end{aligned}
$$

where $N_{L}$ is the number of lines. As a consequence, for large $\kappa d$ the lowest correction to the mean energy is indeed quadratic in $\Delta \rho_{j}$, which is proportional to $A$.

For small $\kappa d$, neighboring lines strongly interact and do not slide past each other. The decomposition of the total interaction energy into contributions from single lines (eq. (4)) is no longer possible and, therefore, the lowest correction of the energy with respect to changing disorder is linear in $A$.

In summary, the mean interaction energy as a function of $A$ is a good indicator of how important the interactions between neighboring lines are. For small $\kappa d$, neighboring lines strongly interact and to lowest order the energy changes linearly in $A$. For large $\kappa d$, neighboring lines are decoupled from each other and the energy is quadratic in $A$.

We report a further interesting observation. For intermediate $\kappa d$ a transition from a solid to a fluid phase occurs with increasing $A$. In particular, in the range of $\kappa d$ from about 10.1 to 12.5 , the system is solid for $A=0$ but melts into a fluid as soon as disorder is introduced $(A>0)$, as we will show in sects. 5 and 6 . Within or close to this range of $\kappa d$, the interaction energy for $A=0$ differs from the extrapolated value $E(A \rightarrow 0)$ and, therefore, $c>1$ in fig. 4(b). So, the mean interaction energy of a crystal is not the same as the energy of a disordered system in the limit of vanishing disorder.

\section{Dynamical properties}

In fig. 3 the first maximum $S_{\max }$ in the structure factor indicated a phase transition from a solid to a fluid phase with increasing $\kappa d$ when $A$ was small. However, for large $A$ it does not differentiate between both phases. Major differences become only visible in dynamical properties. In particular, the system becomes fluid as soon as neighboring lines are able to slide past each other.
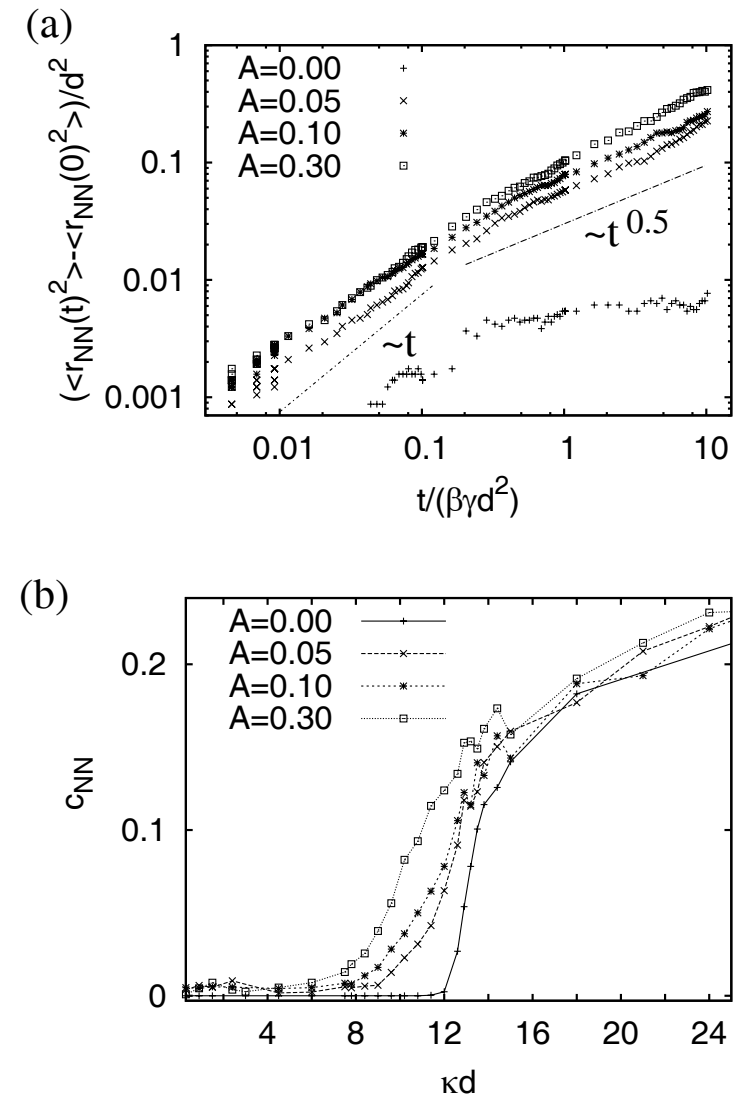

Fig. 5. (a) Mean-square distance between nearest neighbors plotted against time for an inverse screening length $\kappa d=12$ and different values of the control parameter $A$. (b) Prefactor $c_{N N}$ determined from a fit of the time dependence of the nearest-neighbor distance to the function $\left(\left\langle r_{N N}(t)^{2}\right\rangle-\right.$ $\left.\left\langle r_{N N}(0)^{2}\right\rangle\right) / d^{2}=c_{N N}\left[t /\left(\beta \gamma d^{2}\right)\right]^{0.5} \cdot c_{N N}$ is plotted against the inverse screening length $\kappa d$ for different values of the control parameter $A$. The system is solid for $c_{N N}=0$ and fluid for $c_{N N}>0$.

In order to determine the phase boundary between the the disordered solid and fluid phase for all values of $A$, we studied the relative motion of two colloids from neighboring lines that started as nearest neighbors at time $t=0$. Figure 5(a) shows the temporal evolution of their meansquare distance $\left\langle r_{N N}(t)^{2}\right\rangle$. If $\left\langle r_{N N}(t)^{2}\right\rangle$ assumes a constant value for $t \rightarrow \infty$, colloids on neighboring lines cannot pass each other. They are caught in a cage formed by their immediate neighbors and, therefore, their nearest neighbors do not change in time. This behavior is the signature of a solid phase. In fig. $5(\mathrm{a})$, where $\left\langle r_{N N}(t)^{2}\right\rangle-\left\langle r_{N N}(0)^{2}\right\rangle$ is plotted for an intermediate value $\kappa d=12$, the only solid is the crystalline phase at $A=0$. For all non-zero $A,\left\langle r_{N N}(t)^{2}\right\rangle$ grows continuously in time since colloids on neighboring lines can pass each other. This is the signature of the fluid phase. We observe that colloids with respect to their reference particles first display normal diffusion $\left(\left\langle r_{N N}(t)^{2}\right\rangle-\left\langle r_{N N}(0)^{2}\right\rangle \propto t\right)$. However, colloids on the same line cannot pass each other and they ultimately have to show single-file diffusion, where the mean-square displacement only increases proportional to $t^{0.5}[26-28]$. The 


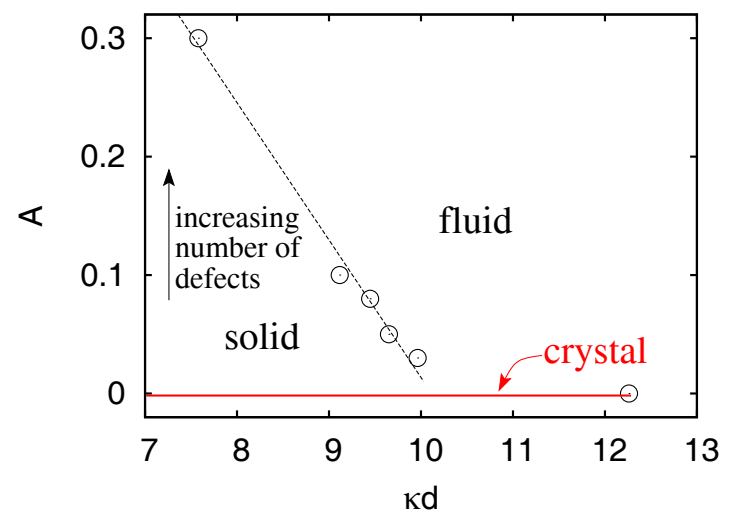

Fig. 6. Phase diagram depending on the inverse screening length $\kappa d$ and the control parameter $A$. The points indicating the phase boundary are the values where the dynamic parameter $c_{N N}$ shown in fig. $5(\mathrm{~b})$ is 0.015 . It seems that the phase boundary is discontinuous for $A \rightarrow 0$. The line is a linear fit to the points for $A>0$.

same also applies to the reference particles in our system. As a consequence, at later times the mean-square distance $\left\langle r_{N N}(t)^{2}\right\rangle-\left\langle r_{N N}(0)^{2}\right\rangle$ in fig. 5(a) passes over to a regime where it grows proportional to $t^{0.5}$. For various parameters $\kappa d$ and $A$, we fitted $\left(\left\langle r_{N N}(t)^{2}\right\rangle-\left\langle r_{N N}(0)^{2}\right\rangle\right) / d^{2}=$ $c_{N N}\left[t /\left(\beta \gamma d^{2}\right)\right]^{0.5}$ to our data and determined the prefactor $c_{N N}$. In a solid $c_{N N}$ is zero, whereas $c_{N N}>0$ in a fluid. We plot $c_{N N}$ in fig. 5(b) as a function of $\kappa d$ for different values of $A$. From this plot, we can then observe the transition from a solid phase at small $\kappa d$ to a fluid at large $\kappa d$.

\section{Phase diagram}

The circles in the phase diagram of fig. 6 indicate where $c_{N N}$ as a function of $\kappa d$ changes from 0 in the frustrated solid phase to a value $c_{N N}>0$ in the fluid. For increasing $A$, the phase transition boundary moves to smaller values of $\kappa d$. Therefore, a disordered phase with larger values of $A$ can be fluidized more easily than a system with less disorder. Interestingly, a linear extrapolation of the phase boundary to $A=0$ gives an inverse screening length $\kappa d \approx 10.1$. However, this does not agree with the crystalto-fluid transition at $A=0$ that occurs at $\kappa d=12.5$. This implies that the phase boundary is discontinuous in agreement with the strong discontinuity of the mean interaction energy reported in sect. 5 in the same $\kappa d$ range.

\section{Summary and outlook}

We have introduced a model system that allows to control disorder continuously so that it crosses over from an ordered crystal into a geometrically frustrated state. The first peak in the structure factor reveals a solid-to-fluid transition in the frustrated phase but only when disorder is sufficiently small. In order to localize the transition and to determine it also for large disorder, we have studied the temporal evolution of the mean-square distance of nearest neighbors on neighboring lines. The transition to the fluid phase occurs when the lines start to slide past each other. This enables us to localize solid and fluid phases in a phase diagram with the degree of disorder and the inverse screening length of the electrostatic interaction as control parameters.

A determining feature of our system is the occurrence of discontinuities when disorder is tuned to zero. For example, the peak height of the pair correlation function in the solid phase does not extrapolate to the value of the perfect crystal. Similarly, the mean interation energy and the screening length at which the solid-fluid transition occurs seem to be discontinuous when the limit of zero disorder is approached. In the limit of infinite system size, it is possible to observe frustrated states that are arbitrarily close to a crystal. Further investigations of such states close to zero disorder should lead to a deeper understanding of how ordered and disordered systems differ.

Among further properties that might be interesting to study in the future are the degeneracy of disordered ground states and the phonon spectrum and eigenmodes that are often employed to characterize glassy or jammed systems (cf., e.g., $[29,30])$. With our model system it is possible to determine how these properties change if the crystalline state is approached from a disordered solid phase. Especially, it will be interesting and important for the characterization of disordered systems to determine and understand which quantities change discontinuously at the transition into the ordered state.

We would like to thank G. Alexander, C. Bechinger, W. Ellenbroek, S. Matsumoto, J. Mikhael, and J. Roth for helpful discussions. We acknowledge financial support from the Deutsche Forschungsgemeinschaft under Grant No. RO 924/5-1. M.S. was supported by the German Academic Exchange Service within the postdoc program.

Open Access This article is distributed under the terms of the Creative Commons Attribution Noncommercial License which permits any noncommercial use, distribution, and reproduction in any medium, provided the original author(s) and source are credited.

\section{References}

1. R. Moessner, Can. J. Phys. 79, 1283 (2001).

2. N. Choudhury, L. Walizer, S. Lisenkov, L. Bellaiche, Nature 470, 513 (2011).

3. L. Pauling, J. Am. Chem. Soc. 57, 2680 (1935).

4. A.R. Ramirez, Nature 421, 483 (2003).

5. P.W. Anderson, Science 235, 1196 (1987).

6. G.H. Wannier, Phys. Rev. 79, 357 (1950).

7. Y. Han, Y. Shokef, A.M. Alsayed, P. Yunker, T.C. Lubensky, A.G. Yodh, Nature 456, 898 (2008).

8. A. Libál, C. Reichhardt, C.J. Olson Reichhardt, Phys. Rev. Lett. 97, 228302 (2006).

9. D.R. Nelson, Phys. Rev. Lett. 50, 982 (1983). 
10. C.D. Modes, R.D. Kamien, Phys. Rev. E 77, 041125 (2008).

11. C. Das, H.R. Krishnamurthy, Phys. Rev. B 58, R5889 (1998).

12. M. Schmiedeberg, J. Roth, H. Stark, Phys. Rev. Lett. 97, 158304 (2006).

13. L.S. Levitov, Phys. Rev. Lett. 66, 224 (1991).

14. D.J. Bishop, P.L. Gammel, D.A. Huse, C.A. Murray, Science 255, 165 (1992).

15. Q. Lu, C.J. Olson Reichhardt, C. Reichhardt, Phys. Rev. B 75, 054502 (2007).

16. A. Chowdhury, B.J. Ackerson, N.A. Clark, Phys. Rev. Lett. 55, 833 (1985).

17. Q.-H. Wei, C. Bechinger, D. Rudhardt, P. Leiderer, Phys. Rev. Lett. 81, 2606 (1998).

18. J. Chakrabarti, H.R. Krishnamurthy, A.K. Sood, S. Sengupta, Phys. Rev. Lett. 75, 2232 (1995).

19. C. Bechinger, M. Brunner, P. Leiderer, Phys. Rev. Lett. 86, 930 (2001).
20. E. Frey, D.R. Nelson, L. Radzihovsky, Phys. Rev. Lett. 83, 2977 (1999).

21. L. Radzihovsky, E. Frey, D.R. Nelson, Phys. Rev. E 63, 031503 (2001).

22. J. Hu, R.M. Westervelt, Phys. Rev. B 55, 771 (1997).

23. J. Baumgartl, M. Brunner, C. Bechinger, Phys. Rev. Lett. 93, 168301 (2004).

24. B.V. Derjaguin, L. Landau, Acta Physicochim. (USSR) 14, 633 (1941).

25. E.J. Verwey, J.T.G. Overbeek, Theory of the Stability of Lyophobic Colloids (Elsevier, Amsterdam, 1948).

26. D.G. Levitt, Phys. Rev. A 8, 3050 (1973)

27. M. Kollmann, Phys. Rev. Lett. 90, 180602 (2003).

28. Q.-H. Wei, C. Bechinger, P. Leiderer, Science 287, 625 (2004).

29. A. Ghosh, V.K. Chikkadi, P. Schall, J. Kurchan, D. Bonn, Phys. Rev. Lett. 104, 248305 (2010).

30. K. Chen, W.G. Ellenbroek, Z. Zhang, D.T.N. Chen, P. Yunker, S. Henkes, C. Brito, O. Dauchot, W. van Saarloos, A.J. Liu, A.G. Yodh, Phys. Rev. Lett. 105, 025501 (2010). 